Please quote as: Schöbel, S.; Janson, A. \& Mishra, A. N. (2019): A Configurational View on Avatar Design - The Role of Emotional Attachment, Satisfaction, and Cognitive Load in Digital Learning. In: International Conference on Information Systems (ICIS). 


\title{
A Configurational View on Avatar Design - The Role of Emotional Attachment, Satisfaction, and Cognitive Load in Digital Learning
}

\author{
Completed Research Paper
}

\author{
Sofia Schöbel \\ University of Kassel \\ Information Systems \\ 34121 Kassel, Germany \\ sofia.schoebel@uni-kassel.de
}

\author{
Andreas Janson \\ University of Kassel \\ Information Systems \\ 34121 Kassel, Germany \\ andreas.janson@uni-kassel.de
}

\author{
Abhay Nath Mishra \\ Iowa State University \\ Information Systems and Business Analytics \\ Ames, Iowa 50011, United States of America \\ abhay@iastate.edu
}

\begin{abstract}
In online learning settings interactive and meaningful feedback is becoming increasingly important. However, feedback from teachers is oftentimes missing in online learning settings. To overcome challenges that arise from the missing representation of teachers, our study analyzes the relevance of avatar designs in learning settings. We therefore rely on avatars as game design elements and analyze how their design can influence emotional attachment, learning process satisfaction, and extraneous cognitive load in learning. To achieve our goal, we conduct a qualitative comparative analysis with 998 datasets that were collected in a $2 \times 2 \times 2$ pre-post online experiment that was developed to train participants in learning functions in Excel. Our results indicate that interaction, familiarity, motivation, and aesthetic experiences are important configurations for avatars that are used in learning. We contribute to different streams of theory such as self-expansion and guide practitioners by providing implications about how to create meaningful avatar designs for learning applications.
\end{abstract}

Keywords: Avatars, Gamification, Qualitative Comparative Analysis, Learning, Emotional Attachment, Satisfaction, Cognitive Load

\section{Introduction}

In private life as well as in work routines, digital learning is becoming increasingly relevant and important due to a proliferating need of upskilling (Bughin et al. 2018). It is well established in the learning literature that direct and individual feedback is one of the most critical drivers influencing learner reaction and behavior and it is necessary to acquire complex cognitive skills (Shute 2008). In a traditional classroom setting, teachers can provide feedback to students through direct and immediate interactions to better support their needs (Means et al. 2009). Such opportunities, however, are limited in online self-learning settings, such as massive open online courses or learning management systems (LMS). In fact, there is a 
large number and variety of digital learning environments where the assistance of a teacher or a trainer is not immediately available (Means et al. 2009).

The lack of a physical presence of a teacher in digital learning environments makes it more challenging to provide helpful, engaging, and motivating feedback to learners about their learning progress (Burgers et al. 2015). An approach to support users with teacher-based feedback is to use an avatar. Avatars are used in several different contexts. They can be used as pedagogical agents (Pérez-Marín and Pascual-Nieto 2013), in combination with smart personal assistants (Pfeuffer et al. 2019), or in the context of games and gamification (Chini et al. 2016; Kim et al. 2007). Besides analyzing the role and meaning of avatars, one of these concepts focusses on the meaning of engagement and motivation in relation to avatars: the concept of gamification. Gamification is about using game-like elements such as avatars in non-entertainmentbased contexts (Deterding et al. 2011). In gamification two kinds of avatars can be used. Avatars can be either used to represent a user or to guide a user (Schöbel and Janson 2018).

Regarding the use of individual elements such as an avatar, research still needs to better understand the relevance of each individual element for different kinds of contexts (Seaborn and Fels 2015). In addition, most studies that focus on gamification try to understand its effects on behavioral outcomes that are oftentimes controversial and do not show direct effects (Super et al. 2019). Referring to this, it is critical to take into consideration the purpose for which an avatar will be used while identifying the most effective design combinations (Mull et al. 2015; Schmidt-Kraepelin et al. 2018; Scott et al. 2014). In digital learning, it is important to analyze in more detail if and how an emotional bond forms between a learner and an avatar (Thomson et al. 2005). Designing an avatar that emotionally attaches learners can influence learning outcomes (Scaife and Rogers 2001; Witmer and Singer 1998). Additionally, affective outcomes in learning, such as satisfaction, are important because these may influence the perception of learners of being able to achieve success and generate positive feelings towards an achieved outcome, which is normally instantiated by a teacher in a traditional classroom setting (Hui et al. 2008). Therefore, it is important to understand how an avatar design can contribute to the satisfaction of learners (Fisher 2003). Finally, digital learning situations present potential for cognitive overload (Mayer and Moreno 2003). Thus, cognitive load aspects of avatar designs for digital learning solutions should be carefully considered by designing avatars that better assist learners when interacting with the system. In particular, designers should consider if a particular design may force learners to interact with the system in an inefficient way or if the design is inadequate (Steed et al. 2016). To better understand how different configurations of the three abovementioned variables impact learning, our research study focuses on the following research question:

$R Q$ : Which configurations determine emotional attachment, satisfaction with the learning process, and cognitive load outcomes with regard to the use of avatars in digital learning?

To answer this question, we use data from an avatar-based online training and analyze them using a Qualitative Comparative Analysis (QCA) approach (Ragin 2009). QCA is a case-oriented method that analyzes asymmetric and complex causal effects by referring to configurations that lead to an outcome of interest. Such a set-theoretic method is useful to conceptualize cases as combinations of attributes (Fiss 2011). Our study provides several contributions for theory and practice. We contribute to avatar and learning theory by referring to three kernel theories: cognitive load theory, self-expansion theory, and flow theory. Additionally, we can provide guidance in developing avatars for learning purposes and, thus, will be able to help practitioners who need to design avatars for their learning applications.

The remainder of the paper is structured as follows. After motivating our research idea, we will present the conceptual background for our paper by referring to our different dependent variables and the configurations we will analyze. Next, we present the method and experimental procedure we used to collect the data for this study. Afterwards we will present the results and will discuss them in a next step. The paper closes with contributions, future research, limitations, and a conclusion.

\section{Conceptual Background}

Figure 1 provides an overview of the design configurations we focus on in relation to emotional attachment, satisfaction with the learning process, and extraneous cognitive load. 


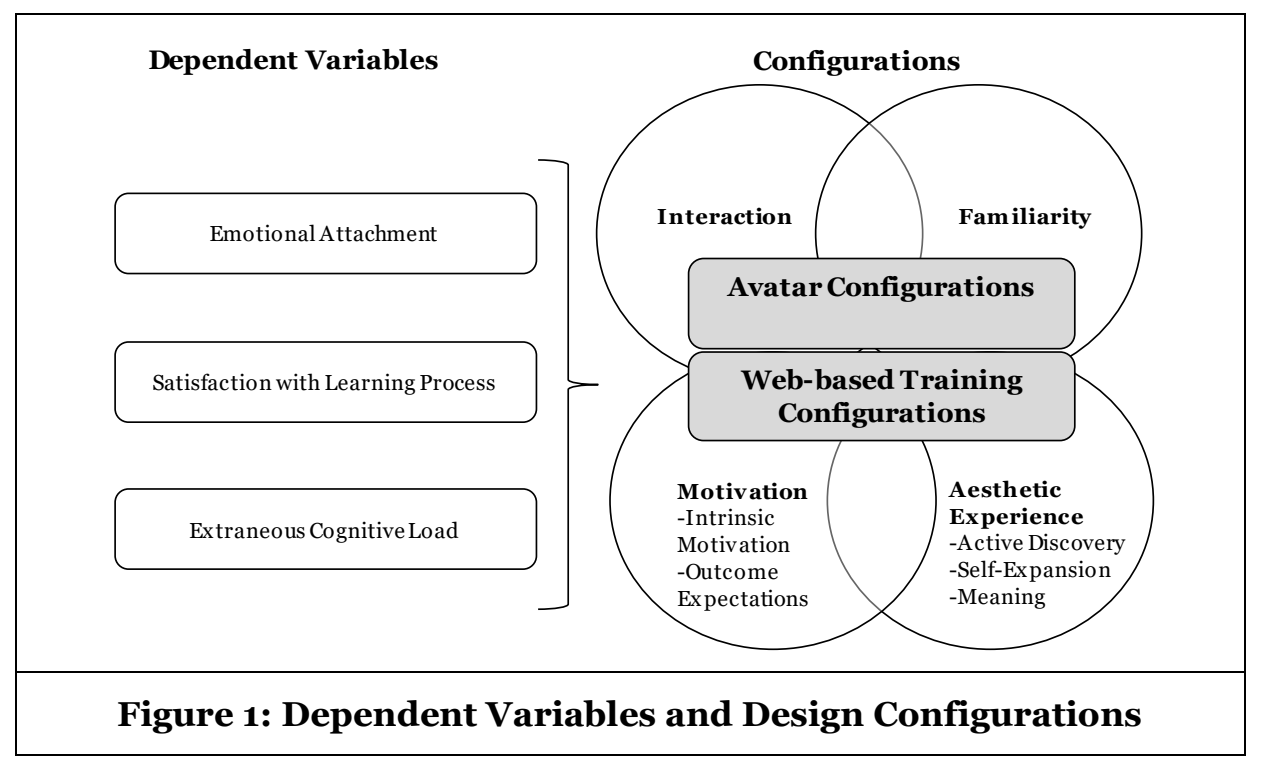

\section{The Role of Emotions, Satisfaction with the Learning Process, and Extraneous Cognitive Load}

An avatar can be defined as a "picture, drawing, or icon that users choose to represent users, typically including images of animals, cartoons, celebrities, or user photographs that embody evil, idiosyncrasy, position, power, and seduction, among other things" (Lee and Kwon 2008, p. 461). Besides using avatars to represent users, they can be used to provide feedback to users (Lee et al. 2013). Avatars can be used for different learning purposes, for example, as pedagogical agents, or as a game element to motivate users. Thus, in the context of learning, avatars can serve a critical function by representing a teacher or trainer (Bedwell et al. 2012). In online learning settings, it becomes more important that learners develop a positive feeling about their virtual teacher by feeling closer to something or someone (Aron et al. 2006). This kind of positive feeling can be described as emotional attachment and can be described as "an emotion-laden, target specific bond between a person and a specific object" (Thomson et al. 2005, p. 77). In online learning settings, where teachers are oftentimes absent, avatars can fulfill the role of a teacher (Christy and Fox 2014; Hoffner and Buchanan 2005). In an online learning environment, avatars have to be designed to support the creation of an emotional bond, which in turn creates a feeling of trust and being supported (Hazan and Zeifman 2008; Mattingly and Lewandowski 2013). Emotional attachment is highly relevant in learning because it supports a person's affection for a specific object (or avatar), which can result in an intention to use an IS more regularly (Suh et al. 2011).

A second aspect that is important in the design of avatars is the affect they engender in users. In this study, we focus on the satisfaction of learners with the learning process. Satisfaction with the learning process can be characterized as an affective learning outcome that "focuses on enhancing the level to which the learner is immersed in the subject" (Gupta and Bostrom 2009, p. 692). Satisfaction with the learning process can be described as the positive feelings of learners related to their performance and related to affective outcomes of learning (Gupta et al. 2010). In a classroom setting this kind of feeling is typically triggered by a teacher who can support learners in improving their performance because satisfaction is higher when users believe the learning and teaching system they use meets their information requirements (Eom 2014). To fulfill this role in an online setting, avatars should be based on creating positive feelings in learning so that learners are more satisfied with their learning process. This feeling of being satisfied with the learning process can be achieved by informing learners how well they have performed and by interacting with them (Ferguson and DeFelice 2010; Fisher 2003). Thus, if an avatar in learning can show how well learners have performed, it can have positive effects on their learning process satisfaction. This in turn will make learners want to continue with their learning process (Ferguson and DeFelice 2010).

Finally, an avatar facilitates learning by interacting with a user during a learning process. Therefore, cognitive load should be considered when designing avatars because online learning situations provide potential for cognitive overload (Mayer and Moreno 2003). Cognitive load is most often considered when 
researching IS, technology-mediated learning, and/or the instructional design of online learning materials. In an online learning environment, where instructors are absent, reducing cognitive load might be even more important because learning happens best when materials are adapted to the human cognitive architecture (Paas et al. 2003). Especially extraneous cognitive load is, among other cognitive load constructs (see Sweller (1994) for more details), of relevance when we consider avatars as an important part of the presentation of online learning materials. Extraneous cognitive load is closely related to the level of usefulness demonstration and a higher level of usefulness demonstration results in a lower level of extraneous cognitive load (Ayres and Youssef 2008). Hence, along with the design of the online training itself, designers should consider a design that supports learners in working and interacting with an avatar (Artino 2008).

\section{Design Configurations for Avatars in Learning}

Two kinds of configurations are important in relation to our three dependent variables: avatar configurations and web-based training configurations. Regarding the avatar design configurations, it is important to consider the familiarity of an avatar in online trainings (Christy and Fox 2016; Mattingly and Lewandowski 2013; Scaife and Rogers 2001). The relevance of familiarity can be explained by selfexpansion theory, which predicts that engaging in an activity with a friend or a known person leads individuals to experience self-expansion (Aron et al. 2006). When using a familiar avatar, learners might be able to develop a relationship and an emotional bond with their avatar. Additionally, with a familiar avatar, learners may experience the feeling of presence, which contributes to more satisfaction (Scaife and Rogers 2001). Extraneous cognitive load is related to the level of usefulness of a demonstration (Ayres and Youssef 2008). Thus, with a familiar avatar it becomes easier for learners to focus on the most important aspects of a training. Another important aspect regarding avatar configuration is the interaction of an avatar with a learner. Interaction can support the development of emotional attachment. Learners who are guided by direct interactions are more likely to have more positive emotional experiences in learning situations (Eccles and Wigfield 1995; Marchand and Gutierrez 2012). Interaction with an avatar is also important to keep learners satisfied. By involving an avatar in an online training, learners have the feeling of being part of a conversation; they feel supported and more satisfied with their learning process (Paas et al. 2003; Van Gerven, et al. 2002). Lastly, interaction is important in terms of avoiding cognitive overload. Learners in multimedia environments experience a cognitive overload when dealing with the complexity of textual and instructional presentations (Paas et al. 2003). Therefore, an avatar should interact with a learner to help them to focus on the most important issues.

Besides configurating an avatar, it is important to configurate the web-based training as well. These configurations are also important to better understand how an avatar can contribute to better learning success. We derived two web-based training configurations: motivation and aesthetic experience. Motivation can be classified into intrinsic and extrinsic motivation (Pintrich 2003). Intrinsic motivation is connected to one's inherent pleasure and interest in the activity (Noels 2001). Extrinsic motivation or outcome expectations is about the judgement of consequences resulting from a behavior (Bandura and Jourden 1991; Gupta and Bostrom 2013). In line with the embedded avatar, the web-based training design (for which the avatar is one component) should be motivating in both ways, addressing intrinsic motivation as well as supporting outcome expectations. An avatar that motivates can support the development of an emotional relationship with a teacher, can reduce cognitive load, and supports learning process satisfaction (Poonam 1997). Aesthetic experience can be described as a state of mind in which a learner's focus is given to an intentional field of actions to accomplish a desired outcome (Suh et al. 2017). Three components are important regarding aesthetic experience: meaning (MEA), active discovery (ACD), and self-expansion (SEP) (Suh et al. 2017). MEA is about the extent to which a learner understands the meaning of an activity. SEP is about a learner's sense of self by knowing things or broadening their perspective and experiencing personal growth. Finally, ACD is important to support learners in actively seeking answers or resolutions to cognitive challenges to achieve their personal goals. Overall, the aesthetic experience contributes to the development of an emotional bond, the reduction of cognitive load, and a higher learning process satisfaction (Jia et al. 2016). Therefore, avatars in learning should facilitate learning to better demonstrate the meaning of the most important aspects they have to learn. Having an avatar that supports learners in actively discovering learning materials, the learner will develop a more stable bound to the avatar (Derrick et al. 2011). Finally, SEP in traditional classroom settings can be developed by a teacher who directly gives 
feedback to a learner (Mayer and Moreno 2003; Means et al. 2009). In online learning settings, an avatar should fulfill this function by giving learners the feeling of being accompanied.

In relation to our three dependent variables, we should consider that learners differ in terms of which avatar configuration they like. Therefore, we consider prior knowledge and gender in relation to our dependent variables. Referring to prior knowledge, experts might be able to process information faster than novices, who need more guidance (Felder and Brent 2005; Hailikari et al. 2008). Therefore, avatar design configurations (especially interaction) might differ regarding cognitive load. In addition, we will consider gender with regard to avatar designs. Women and men might differ regarding their emotional bond towards avatars. Women might be more attached to a familiar avatar than men because they are more interpersonally oriented to something or someone than men (Venkatesh and Morris 2000). Furthermore, females might be more interested in familiar avatars because they are more interested in actions that involve social interactions and have a greater awareness of other's feelings (Greenberg et al. 2010; Sun and Ping 2006). In summary, we found support that gender and prior knowledge might be important when considering emotional attachment and cognitive load. A more detailed analysis of subsamples regarding satisfaction is not part of this study but relevant for future research.

\section{Research Methodology}

\section{Study Design, Manipulations, and Measurements}

To collect our data, we developed an online 2x2x2 pre-post-test, between-subject experiment with an additional control group. We considered female as well as male avatars to analyze if there is a difference between their impacts on outcomes of interest. An example of the training is given in Figure 2.

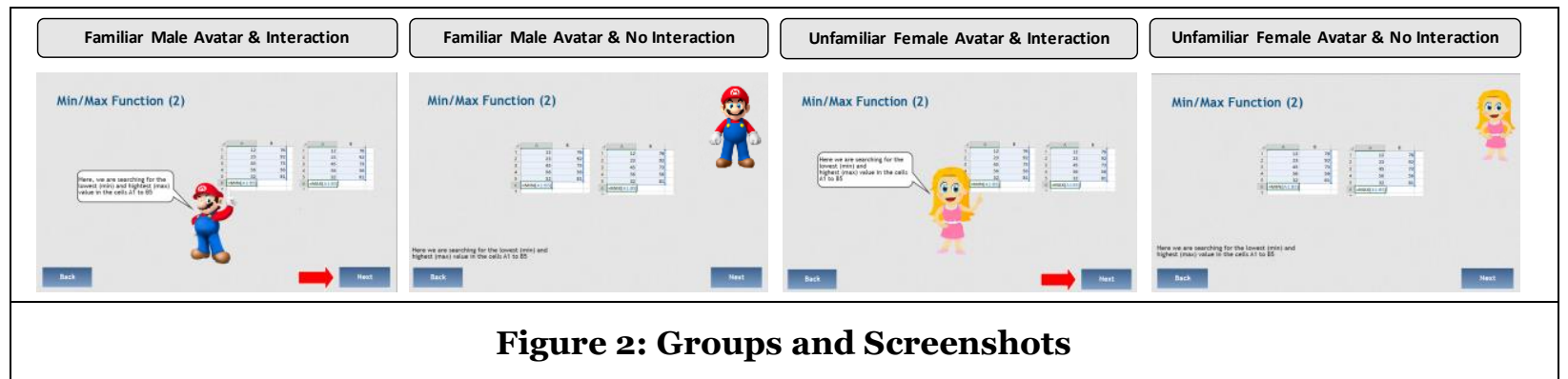

To analyze the familiarity, we used Super Mario as the familiar male avatar and Princess Peach as the familiar female avatar. We used an unfamiliar male avatar, which we have already implemented and evaluated in another learning application (Ernst et al. 2016). Following the clothes and hair color of Princess Peach, we also adapted the unknown female avatar. With regard to the interaction between the avatars and learners, direct instructions were given by the avatar in a speech bubble and with gestures. Indirect instructions were placed near a task. One group without an avatar was considered to determine the overall influence of avatars. Motivation and flow were covered by the overall presence of avatars as well as by the implemented interactions and were measured in a post-test after the participants had completed the training.

The learning goal of our online training was to train the participants in Microsoft Excel. We decided to use Excel because Excel has also been used in previous studies of online learning, thus making results of the study more comparable to the existing literature (Gupta and Bostrom 2013). We used a five item, bipolar 7-point Likert scale from Gupta and Bostrom (2013) to measure learning process satisfaction. Emotional attachment was measured with 10 items and a 7-point Likert scale (Thomson et al. 2005). Extraneous cognitive load was measured with 3 items and a 7-point Likert scale (Ayres and Youssef 2008). We measured intrinsic motivation (three items) and outcome expectations (seven items) with a 7-point Likert scale (Gupta and Bostrom 2013). SEP, MEA, and ACD were measured with three items, each on a 7-point Likert scale (Mattingly and Lewandowski 2013; Suh et al. 2017).The participants' prior knowledge was measured on a 7-point Likert scale with two items asking them about their experience with Excel in general and their experience with functions in Excel. 


\section{Data Collection and Measurement Validation}

To assess the quality of our survey and the online training, we conducted a pre-test. We asked ten graduated students to test our Excel Training. Overall, apart from some typing mistakes, the students stated that our online training is understandable. After applying minor changes to the wording and adding information about the structure in the beginning of our online training, we collected the data via e-mail. We asked students for participation. Student samples have been used in the prior literature for evaluating online learning models or training interventions (Gupta and Bostrom 2013; Santhanam et al. 2016), thereby enabling us to compare our results. We used a javascript-based randomization procedure to randomly assign participants to one of our nine groups, and we assured by setting mandatory cookies that participants could not change groups or do the training more than once. Students were recruited via e-mail. We sent five mails in total: The first one as an initial invitation, the other ones as friendly reminders. Students from different subjects, different ages, and degrees participated. The mail was sent to all students of our university. The students did get course credits for completing the web-based training as well as the postsurvey. They had to enter their mail address at the end of the survey so that we could check if they completed the training and the survey. In addition, we included control questions to see if the participants concentrated during the training. The training started with a background survey that was used to evaluate demographic data such as age and gender. Additionally, we evaluated the participants' prior knowledge of Excel. Afterwards, the participants were randomly assigned to one of the nine groups. In a last step, a questionnaire was included to measure our dependent variables and conditions. On average, the training lasted between 50 and 60 minutes. In total, we obtained 998 complete responses for our analysis. Table 1 provides an overview of the demographic data.

\begin{tabular}{|c|c|c|c|}
\hline \multicolumn{4}{|c|}{ Table 1: Demographic Data } \\
\hline Group & Number & Age & Gender \\
\hline Control Group (C) & 101 & Mean: 25.52 (Min: 18, Max: 54) & $\mathrm{F}: 41, \mathrm{M}: 60$ \\
\hline Male, Known, Direct (MKD) & 103 & Mean: 24.77 (Min: 19, Max:38) & F: $45, M: 58$ \\
\hline Female, Known, Direct (FKD) & 106 & Mean: 25.49 (Min: 19, Max:63) & $\mathrm{F}: 63, \mathrm{M}: 43$ \\
\hline Male, Known, Indirect (MKI) & 102 & Mean: 25.05 (Min: 18, Max:39) & $\mathrm{F}: 52, \mathrm{M}: 50$ \\
\hline Female, Known, Indirect (FKI) & 128 & Mean: 25.22 (Min: 18, Max:39) & $\mathrm{F}: 79, \mathrm{M}: 49$ \\
\hline Male, Unknown, Direct (MUD) & 101 & Mean: 24.62 (Min: 17, Max: 40) & $\mathrm{F}: 58, \mathrm{M}: 43$ \\
\hline Female, Unknown, Direct (FUD) & 118 & Mean: 25.14 (Min: 18, Max: 51) & $\mathrm{F}: 64, \mathrm{M}: 54$ \\
\hline Male, Unknown, Indirect (MUI) & 125 & Mean: 25.04 (Min: 18, Max: 49) & $\mathrm{F}: 54, \mathrm{M}: 71$ \\
\hline Female, Unknown, Indirect (FUI) & 114 & Mean: 25.07 (Min: 17, Max: 39) & $\mathrm{F}: 52, \mathrm{M}: 62$ \\
\hline Total & 998 & Mean: 25.10 & $F: 431, M: 567$ \\
\hline
\end{tabular}

Table 1: Demographic Data

To ensure validity, reliability, as well as rigor of our measurement instruments, we calculated the loadings, and Cronbach's alpha (above 0,707 ) for each construct. Confirmatory factor analysis indicated high factor loadings (all above 0,7) and a fulfillment of the Fornell Larcker criterion (Fornell and Larcker 1981). Table 2 shows the results.

\begin{tabular}{|l|l|l|}
\hline \multicolumn{3}{|c|}{ Table 2: Measurement Scales } \\
\hline \multicolumn{2}{|l|}{ Factor Loadings (>0.7) } & Cronbach's Alpha (>0.707) \\
\hline Construct & \multicolumn{2}{|l|}{} \\
\hline Outcome Variable & $0.770-0.817$ & 0.899 \\
\hline Emotional Attachment & $0.766-0.901$ & 0.883 \\
\hline Satisfaction with Learning Process & $0.737-0.949$ & 0.807 \\
\hline Cognitive Load & $0.872-0.906$ & 0.875 \\
\hline Causal Conditions & $0.878-0.912$ & 0.886 \\
\hline Meaning (MEA) & $0.872-0.920$ & 0.880 \\
\hline Active Discovery (ACD) & $0.771-0.914$ & 0.938 \\
\hline Self-Expression (SEP) & $0.935-0.966$ & 0.947 \\
\hline Outcome Expectations (OE) & Intrinsic Motivation (IM) &
\end{tabular}

Table 2: Measurement Scales 


\section{Fuzzy-set QCA and Crisp-set QCA}

The collected data were used for our QCA analysis. In QCA two different analyses are performed to analyze data, a fuzzy set and a crisp set (Ragin 2009). For our research, we use a fuzzy set QCA (FsQCA) for emotional attachment, satisfaction with the learning process, extraneous cognitive load, motivation, and aesthetic experience. We used a crisp set for interaction and familiarity. FsQCA emphasizes the effects of the overall analysis rather than its pieces. Thus, FsQCA evaluates the predictive power of different configurations of conditions (our avatar designs) based on their measurements, consistency values, and coverage values (Fiss 2011). Consistency values can be interpreted like correlations and they describe the degree to which specific cases share a certain combination of conditions in relation to an outcome (Ragin 2009). For our analysis a case is represented by the answers given to our scales by our participants. A case also reflects if a participant was working with a direct or indirect avatar and a familiar or unknown one by coding them with 1 for familiar and interactive and with o for unknown and not interactive. Coverage values can be interpreted like R-square values and indicate the degree to which a configuration explains an outcome. For FsQCA two different coverage values are of relevance: the raw coverage and the unique coverage. Raw coverage values indicate the chance that a case in a data set can be observed; the unique coverage value reflects the percentage of cases that show a membership in the configuration but not in any other configuration (Ragin 2009).

Ragin (2009) suggests three steps to conduct a QCA: (1) calibration, (2) construction of truth tables, (3) truth table analysis. First, a data calibration is necessary. QCA works with sets that indicate a membership or a non-membership. To identify which value is a member and which is not, each variable has to be calibrated (Schneider and Wagemann 2012). Fuzzy membership score ranges from o, indicating a nonmembership, and 0.5 , indicating a crossover point, to 1, indicating a full membership (Schneider and Wagemann 2012). In our research we used 7-point Likert scales and used 1 as the minimum value, 4 as the crossover value, and 7 as the maximum value for our Likert scales. In our data set, interaction and familiarity are crisp set values indicating whether they are present (using a 1) or not (using a o). The constructs we used consist of several items. The mean values for each construct were then used for the calibration procedure that is provided in the FsQCA software program and that transfers all values to socalled "fuzzy values". The calibration procedure of the FsQCA software calculates fuzzy set values for each of our constructs based on the membership values we set. In a second step, a so-called truth table is calculated. A truth table provides an overview of all possible configurations of conditions. In general, $2^{\mathrm{k}}$ cases are possible, wheras $\mathrm{k}$ stands for the number of conditions observed. For our paper, we have 7 conditions for emotional attachment, satisfaction, and cognitive load, respectively, and thus 128 possible configurations. For large-scale N QCAs, at least seven conditions are necessary (Greckhammer et al. 2013). The truth table is calculated by using the FsQCA software and is refined in a next step. In this refinement process each possible combination is assessed based on the frequency and consistency of each condition. Frequency is analyzed to assess how many cases have a membership score higher than 0.5 in a configuration in order to evaluate if this configuration needs to be considered in the further analysis. We considered around 95\% of all cases and used a cutoff for the frequency of 3 as suggested by Greckhammer (2013).

In a third and last step, the truth table is analyzed. The approach that is used to analyze the truth table is based on the Quine-McCluskey algorithm. This algorithm calculates combinations of different factors which lead to an outcome by removing factors that are inconsistently present or absent regarding a specific outcome (Fiss 2011). Thus, the algorithm excludes conditions that are not part of a configuration of an outcome. The analysis of the tables is conducted in two stages. First, a parsimonious solution is important for the analysis, which includes all assumptions derived from counterfactuals in contrast to the intermediate solution. Second, an intermediate solution is calculated, which represents a subset of the parsimonious solutions. Both solutions are compared to identify which conditions are core and which are peripheral conditions. The intermediate solutions highlight the consistency and coverage values as well as the different configurations that are relevant for a dependent variable.

\section{Results}

To analyze our data, we follow the recommendations provided by Greckhammer et al. (2013). When analyzing QCA data, it should be considered if researchers are working with large-N QCA or small-N QCA. 
Small-N QCA considers between 12 and 50 cases, and large-N QCA considers more than 50 cases. With 998 cases our study is a large-N QCA study. In addition, the relationship of small-N QCA cases is close, for large$\mathrm{N}$ it is distant. small-N QCAs normally have between 4 and 8 conditions and large-Ns between 6 and twelve. To analyze QCA data, consistency and coverage values are important. In large-N QCAs, consistency values should be above 0.80 . For small-N QCAs consistency values should be 1. Coverage values for large-N QCAs are relatively lower, large coverage is desirable but not necessary (see Table 3 for a summary).

\begin{tabular}{|l|l|l|}
\hline \multicolumn{5}{|c|}{ Table 3: Criteria for small-N QCA and large-N QCA (Greckhammer et al. 2013, p. 54) } \\
\hline Number of Cases & Small-N QCA & Large-N QCA \\
\hline Relationship of Cases & $\begin{array}{l}\text { Close, based on knowledge of each } \\
\text { case }\end{array}$ & $\begin{array}{l}\text { Distant, based on knowledge of } \\
\text { conceptual relationship }\end{array}$ \\
\hline Number of conditions & $4-8$ & $6-12$ \\
\hline Consistency & Should be 1 (o.8 is also acceptable) & $>=0.8$ \\
\hline Coverage & Typically high & Relatively lower \\
\hline
\end{tabular}

Table 3: Criteria for small-N QCAs and large-N QCAs (Greckhammer et al. 2013, p. 54)

We are going to present the results for each dependent variable in the following sections. For each configuration it can be determined if a condition is absent or present. This absence or presence is indicated by different types of circles. Black circles indicate the presence of a condition, crossed-out circles indicate their absence. Large circles indicate a core condition, and small circles indicate peripheral conditions. Blank spaces indicate a condition may be either present or absent. To better guide our analysis and discussion, and to mark the most important results of our analysis, we highlighted some fields in each table. The row with the best configuration of conditions is marked in bold. Conditions that were present in most configurations are marked in grey. Those that are absent in most conditions are marked with a dotted line.

\section{Emotional Attachment}

We started our analysis with analyzing emotional attachment as a dependent variable. The results for the complete dataset can be seen in Table 4 .

\begin{tabular}{|c|c|c|c|c|c|c|c|c|}
\hline \multicolumn{9}{|c|}{ Table 4: Results for Emotional Attachment } \\
\hline \multirow[b]{2}{*}{ Conditions } & \multicolumn{8}{|c|}{ Configurations } \\
\hline & $\mathbf{1}$ & $\mathbf{2}$ & 3 & 4 & 5 & 6 & $\overline{7}$ & 8 \\
\hline \multicolumn{9}{|l|}{ Avatar Design } \\
\hline Interaction & & & $\otimes$ & & & & & \\
\hline \multicolumn{9}{|l|}{ Familiarity } \\
\hline \multicolumn{9}{|l|}{ Aesthetic Experience } \\
\hline \multicolumn{9}{|l|}{ MEA } \\
\hline \multicolumn{9}{|l|}{$\mathrm{ACD}$} \\
\hline \multicolumn{9}{|l|}{ SEP } \\
\hline \multicolumn{9}{|l|}{ Motivation } \\
\hline \multicolumn{9}{|l|}{ IM } \\
\hline \multicolumn{9}{|l|}{$\mathrm{OE}$} \\
\hline Consistency & 0.875 & 0.831 & 0.843 & 0.883 & 0.896 & 0.883 & 0.899 & 0.858 \\
\hline Raw Coverage & 0.407 & 0.093 & 0.133 & 0.092 & 0.180 & 0.092 & 0.168 & 0.085 \\
\hline Unique Coverage & 0.023 & 0.010 & 0.002 & 0.013 & 0.020 & 0.004 & 0.017 & 0.003 \\
\hline \multicolumn{8}{|l|}{ Overall Solution Consistency } & 0.844 \\
\hline Overall Solution Coverage & & & & & & & & 0.501 \\
\hline
\end{tabular}

Table 4: Results for Emotional Attachment

Eight different configurations could be identified. Consistency for configurations ranges from 0.831 to 0.899. Raw coverage ranges from 0.085 to 0.407 . The overall solution consistency indicates these eight 
conditions can result in a high likelihood of emotional attachment with $84 \%$ and the overall solution coverage indicates that the extent to which these eight configurations cover a high likelihood of emotional attachment is $50 \%$ (of all of our cases). The strongest emotional attachment results from the presence of five different conditions: familiarity, MEA, SEP, IE, and OE. In this combination ACD is absent. The most present conditions along all configurations are SEP and IM. Next, we analyzed emotional attachment of females and males, starting with the subsample with females (see Table 5).

\begin{tabular}{|c|c|c|c|c|}
\hline \multicolumn{5}{|c|}{ Table 5: Emotional Attachment Females } \\
\hline \multirow[b]{2}{*}{ Conditions } & \multicolumn{4}{|c|}{ Configurations } \\
\hline & $\mathbf{1}$ & 2 & 3 & 4 \\
\hline \multicolumn{5}{|l|}{ Avatar Design } \\
\hline Interaction & & & $\otimes$ & $\otimes$ \\
\hline \multicolumn{5}{|l|}{ Familiarity } \\
\hline \multicolumn{5}{|l|}{ Aesthetic Experience } \\
\hline \multicolumn{5}{|l|}{ MEA } \\
\hline \multicolumn{5}{|l|}{ ACD } \\
\hline \multicolumn{5}{|l|}{ SEP } \\
\hline \multicolumn{5}{|l|}{ Motivation } \\
\hline \multicolumn{5}{|l|}{ IM } \\
\hline \multicolumn{5}{|l|}{$\mathrm{OE}$} \\
\hline Consistency & 0.848 & 0.810 & 0.812 & 855 \\
\hline Raw Coverage & 0.425 & 0.339 & 0.092 & 105 \\
\hline Unique Coverage & 0.139 & 0.135 & 0.006 & 016 \\
\hline \multicolumn{4}{|l|}{ Overall Solution Consistency } & 0.808 \\
\hline Overall Solution Coverage & & & & 0.594 \\
\hline
\end{tabular}

Table 5: Emotional Attachment Females

The four different configurations result in a high emotional attachment with $80 \%$ and cover $60 \%$ of cases with this outcome. Raw coverage ranges from 0.092 to 0.425 and all consistency values are above o.8.

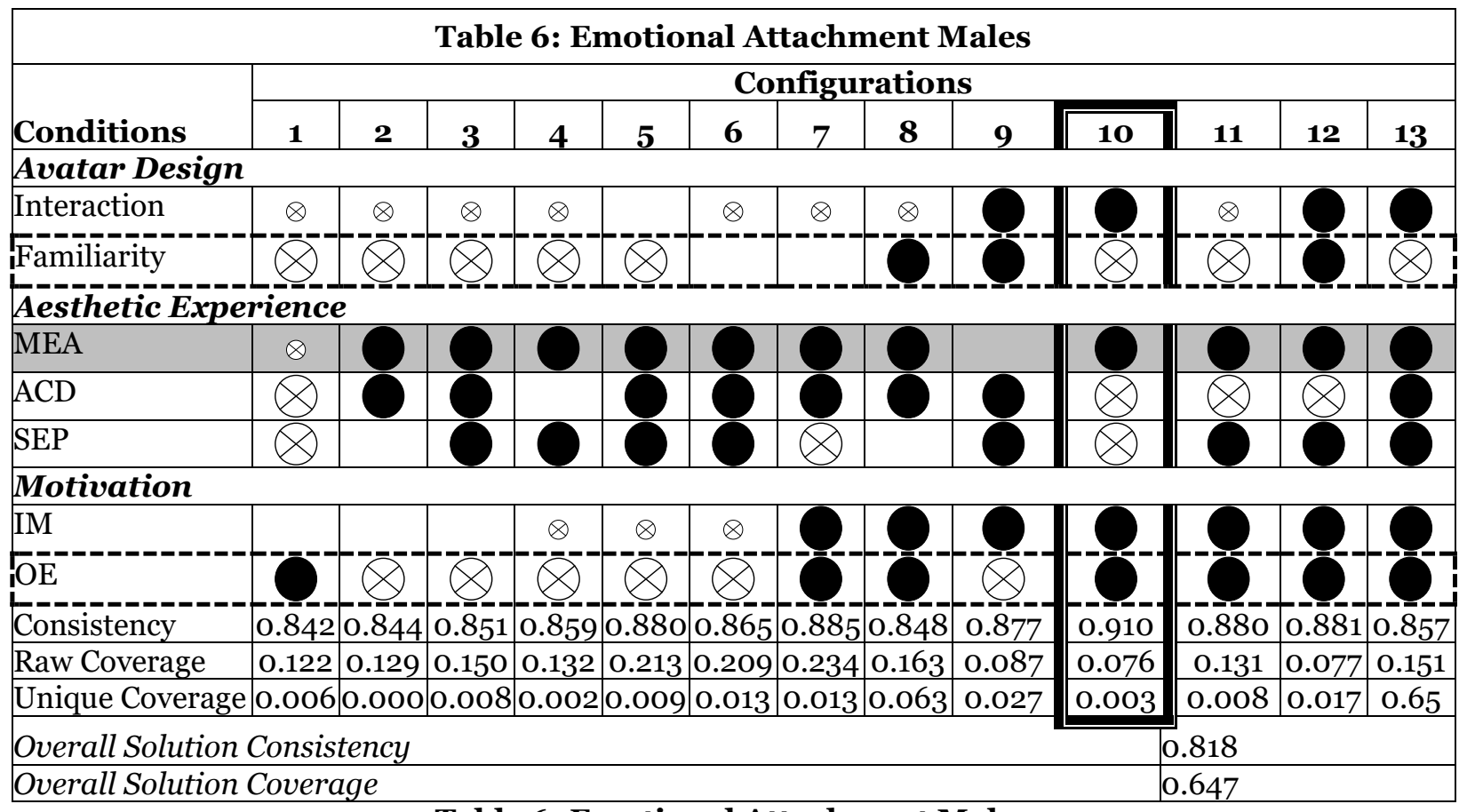

Table 6: Emotional Attachment Males 
To better demonstrate how the results for females and males differ, we present the results for the male participants in Table 6. The thirteen different configurations for the male subsample result in a high emotional attachment of $81 \%$ and a coverage of $64 \%$. Raw coverage ranges from 0.077 to 0.213 . All consistency values are above 0.8 . Based on the consistency values, the strongest emotional attachment of females develops with familiar avatars, in combination with MEA, ACD, IM, and OE. SEP as well as interaction are absent, whereby the strongest emotional attachment of males develops with interactive avatars, in combination with MEA, IM, and OE. SEP, ACD, and familiarity are absent. Next, we will continue with describing the results for satisfaction with the learning process.

\section{Satisfaction}

Besides evaluating configurations for emotional attachment, we assessed how avatars impact learning process satisfaction. The results are presented in Table 7 . These solutions consistently result in a likelihood of satisfaction with $94 \%$ and cover $58 \%$ of cases with this outcome. All consistency values are above 0.8 and raw coverage is between 0.054 to 0.329 . The strongest configuration results from a combination of interaction, MEA, IM, and ACD. The most present conditions are IM and OE. The most absent one is interaction.

\begin{tabular}{|c|c|c|c|c|c|c|c|c|c|}
\hline \multicolumn{10}{|c|}{ Table 7: Results for Satisfaction with the Learning Process } \\
\hline \multirow[b]{2}{*}{ Conditions } & \multicolumn{9}{|c|}{ Configurations } \\
\hline & $\mathbf{1}$ & 2 & 3 & 4 & 5 & 6 & 7 & 8 & 9 \\
\hline \multicolumn{10}{|l|}{ Avatar Design } \\
\hline \multicolumn{10}{|l|}{ Interaction } \\
\hline \multicolumn{10}{|l|}{ Familiarity } \\
\hline \multicolumn{10}{|c|}{ Aesthetic Experience } \\
\hline \multicolumn{10}{|l|}{ MEA } \\
\hline \multicolumn{10}{|l|}{ ACD } \\
\hline \multicolumn{10}{|l|}{ SEP } \\
\hline \multicolumn{10}{|l|}{ Motivation } \\
\hline \multicolumn{10}{|l|}{ IM } \\
\hline \multicolumn{10}{|l|}{$\mathrm{OE}$} \\
\hline Consistency & 0.946 & 0.964 & 0.951 & 0.973 & 0.964 & 0.962 & 0.953 & 0.955 & 0.940 \\
\hline Raw Coverage & 0.236 & 0.329 & 0.363 & 0.076 & 0.151 & 0.206 & 0.054 & 0.074 & 0.220 \\
\hline Unique Coverage & 0.015 & 0.019 & 0.069 & 0.003 & 0.012 & 0.000 & 0.008 & 0.006 & 0.002 \\
\hline \multicolumn{9}{|c|}{ Overall Solution Consistency } & 0.941 \\
\hline \multicolumn{9}{|c|}{ Overall Solution Coverage } & 0.579 \\
\hline
\end{tabular}

Table 7: Results for Satisfaction with the Learning Process

We did not further analyze gender or prior knowledge that is different towards satisfaction with the learning process. We found theoretical support to analyze gender differences for emotional attachment and differences in prior knowledge for extraneous cognitive load deeper. However, a deeper analysis of satisfaction with the learning process could be relevant for future research, which we outline in more detail in the last section of this paper.

\section{Extraneous Cognitive Load}

Next, we present the results for extraneous cognitive load. The results for the complete data set are shown in Table 8. The solution consistency results in a low likelihood of extraneous cognitive overload of $92 \%$ and cover $50 \%$ of all cases that were analyzed. Consistency values are above 0.8 , and raw coverage values are between 0.133 and 0.352. Regarding the consistency values of the individual configurations, a combination of IM and OE, and an absence of ACD and SEP results in 95\% consistency. The most present condition in all configurations is IM. The most absent conditions are interaction and ACD. 


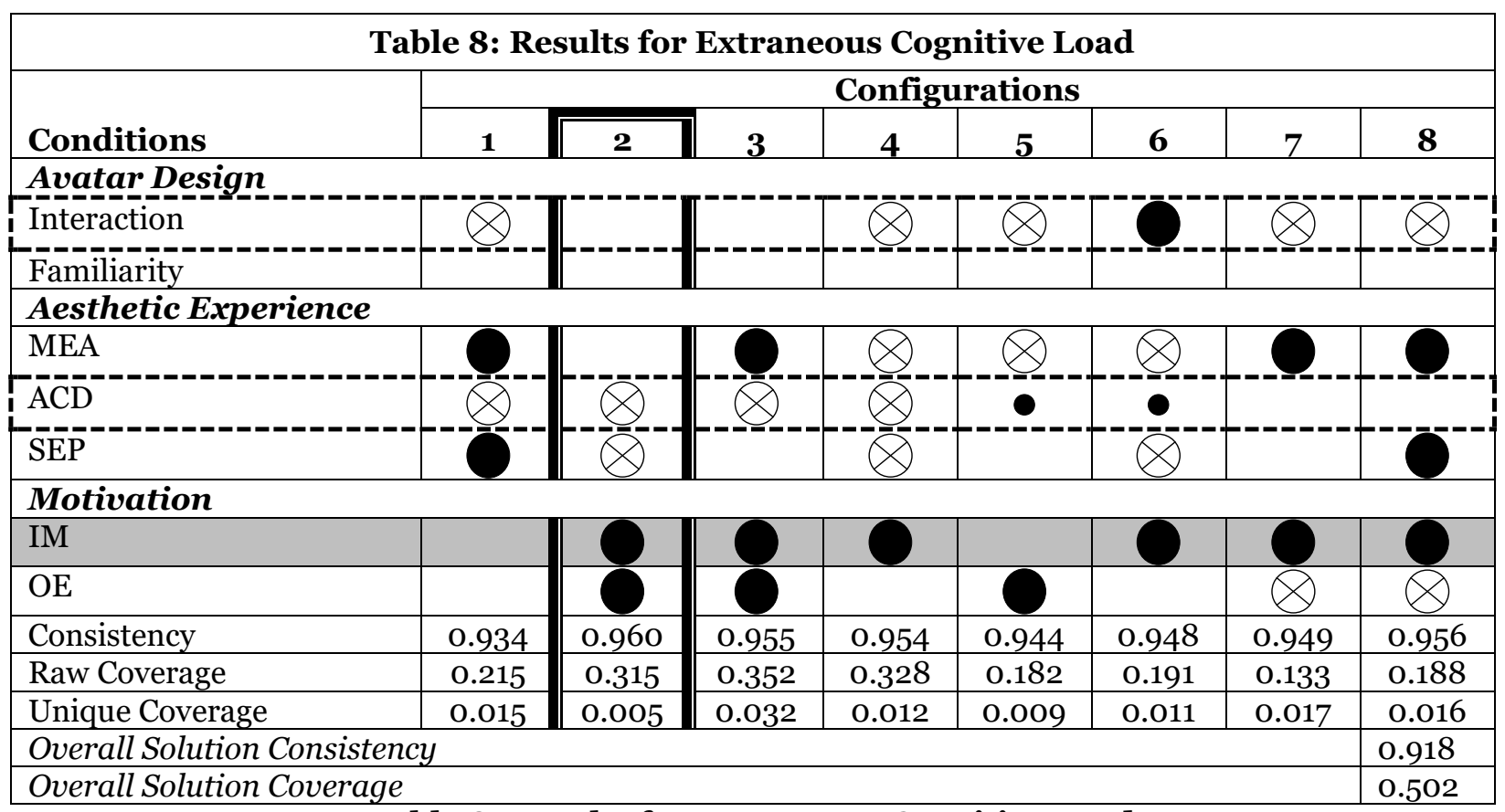

Table 8: Results for Extraneous Cognitive Load

To understand how learners' differ with regard to their extraneous cognitive load, we divided the overall sample into two sub-samples by analyzing Excel experts and novices, starting with experts (see Table 9).

\begin{tabular}{|c|c|c|c|c|c|c|c|c|}
\hline \multicolumn{9}{|c|}{ Table 9: Results for Extraneous Cognitive Load - Experts } \\
\hline \multirow[b]{2}{*}{ Conditions } & \multicolumn{8}{|c|}{ Configurations } \\
\hline & $\mathbf{1}$ & 2 & 3 & 4 & 5 & 6 & 7 & 8 \\
\hline \multicolumn{9}{|l|}{ Avatar Design } \\
\hline Interaction & & & & & $\otimes$ & $\otimes$ & & $\otimes$ \\
\hline \multicolumn{9}{|l|}{ Familiarity } \\
\hline \multicolumn{9}{|c|}{ Aesthetic Experience } \\
\hline \multicolumn{9}{|l|}{ MEA } \\
\hline \multicolumn{9}{|l|}{ ACD } \\
\hline \multicolumn{9}{|l|}{ SEP } \\
\hline \multicolumn{9}{|l|}{ Motivation } \\
\hline \multicolumn{9}{|l|}{ IM } \\
\hline \multicolumn{9}{|l|}{$\mathrm{OE}$} \\
\hline Consistency & 0.961 & 0.954 & 0.969 & 0.968 & 0.964 & 0.953 & 0.955 & 0.964 \\
\hline Raw Coverage & 0.249 & 0.303 & 0.131 & 0.307 & 0.148 & 0.149 & 0.113 & 0.162 \\
\hline Unique Coverage & 0.021 & 0.043 & 0.004 & 0.027 & 0.016 & 0.018 & 0.007 & 0.010 \\
\hline \multicolumn{8}{|c|}{ Overall Solution Consistency } & 0.932 \\
\hline \multicolumn{8}{|c|}{ Overall Solution Coverage } & 0.475 \\
\hline
\end{tabular}

Table 9: Results for Extraneous Cognitive Load - Experts

All eleven configurations that were identified result in a low likelihood of extraneous cognitive overload with $93 \%$ and cover $47 \%$ of all cases. Raw coverage ranges from 0.113 to 0.307 with consistency values all above 0.8. The same analysis was made for the group of novices (see Table 10). Having little experience with Excel results in a low likelihood of extraneous cognitive overload with $85 \%$ and $62 \%$ coverage. All consistency values are above 0.8 and raw consistency ranges from 0.138 to 0.414 . For experts the most promising configuration to reduce extraneous load is to refer to MEA, IM, OE, and an absence of SEP. For novices a combination of ACE, SEP, IM, and OE and the absence of interaction, and MEA results as most 
promising configuration to influence extraneous cognitive load. Regarding the most present and absent conditions, in the data set with experienced learners, ACD, and IM are present in almost all configurations. For the data set with novices, ACD is present in almost all configurations.

\begin{tabular}{|c|c|c|c|c|c|c|c|c|c|c|c|}
\hline & Tal & le 1o: 1 & Results & for $\mathrm{Ex}$ & raneol & s Cogr & tive Lo & $\operatorname{ad}-\mathbf{N}$ & vices & & \\
\hline & & & & & Co & ifigur: & ions & & & & \\
\hline Conditions & $\mathbf{1}$ & 2 & 3 & 4 & 5 & 6 & 7 & 8 & 9 & 10 & 11 \\
\hline Avatar Desig & & & & & & & & & & & \\
\hline Interaction & $\otimes$ & & & & & & $\otimes$ & & & & \\
\hline Familiarity & & & & & & & & & & & \\
\hline Aesthetic Ex & perien & & & & & & & & & & \\
\hline MEA & & & & & $\otimes$ & & & & & & \\
\hline $\mathrm{ACD}$ & & & & & $\otimes$ & & 8 & & & & \\
\hline SEP & & & & & 8 & & 8 & $\otimes$ & & & \\
\hline Motivation & & & & & & & & & & & \\
\hline $\mathrm{IM}$ & & & & & & & & & & & \\
\hline$\overline{\mathrm{OE}}$ & & & $\infty$ & & & $\infty$ & & & & 8 & \\
\hline Consistency & 0.871 & 0.876 & 0.917 & 0.894 & 0.923 & 0.930 & 0.945 & 0.938 & 0.946 & 0.934 & 0.959 \\
\hline Raw Coverage & 0.251 & 0.253 & 0.412 & 0.414 & 0.304 & 0.202 & 0.203 & 0.219 & 0.353 & 0.138 & 0.195 \\
\hline \begin{tabular}{|l|} 
Unique \\
Coverage
\end{tabular} & 0.004 & 0.004 & 0.030 & 0.014 & 0.014 & 0.001 & 0.005 & 0.010 & 0.031 & 0.012 & 0.005 \\
\hline Overall Solutic & n Cons & tency & & & & & & & 0.8 & & \\
\hline Overall Solutic & n Cove & age & & & & & & & 0.61 & & \\
\hline
\end{tabular}

Table 10: Results for Extraneous Cognitive Load - Novices

Having the results of our study in mind, we now will discuss them in more detail in the next section.

\section{Discussion and Contributions}

The results of our paper provide room to discuss them in more detail. The results indicate that we need to rethink the concept of gamification in terms of the oftentimes used one-size-fits-all solutions. There is some support that different designs are necessary to better address behavioral outcomes of users (Santhanam et al. 2016). This is further supported by the recommendation to pay more attention to each individual element used in gamification (Seaborn and Fels 2015). This recommendation can be supported when we look at the results of our study about avatar designs. Female users are emotionally attached to familiar avatars, in contrast to males, who are more interested in having interacting avatars in relation to their emotional attachment. For learners with high prior knowledge about a topic interaction of avatars, extraneous cognitive load is important, whereby learners with low prior knowledge do not need interactive avatars in relation to extraneous cognitive load. Our results indicate that avatars are important to address emotional attachment, satisfaction with the learning process, and extraneous cognitive load. However, our results also demonstrate that for some variables web-based training configurations are even more important. When we look at satisfaction with the learning process, we can see that the most present condition along our configurations is IM, avatar interaction is mostly absent. Similar to this, IM and ACD are important when we look at the configurations of extraneous cognitive load. Thus, we can assume that for both satisfaction with the learning process as well as extraneous cognitive load, it is important to care about motivational as well as aesthetic components. If we take a closer look at OE, we can see it is not that important to develop an emotional attachment because it is absent in most configurations for emotional attachment. So it is up to a system designer to find the right balance between both kinds of motivational orientation, which can be very difficult because intrinsic and extrinsic motivation can be related (Deci et al. 2001). If web-based trainings are not grounded in a motivational design or do not address aesthetic experience (e.g., by better demonstrating the meaning of learning material), we can assume that an avatar would not contribute to any of our outcome variables. On the other hand, if a training supports motivation 
and the aesthetic experience of an avatar, then its design might be effective in creating an emotional bond increasing satisfaction or lowering cognitive load.

Interaction and familiarity are two important constructs to design avatars for learning. However, interaction can result in negative consequences regarding cognitive load, especially when we interact in the area of learning (van Merriënboer and Sweller 2005). Avatar interactions can also be connected to sounds (audible feedback (Thiebes et al. 2014) or further animated movements (Lin et al. 2013)). Due to this reason we kept the interaction of our avatars relatively simple by integrating speech bubbles and gestures. With a more animated or interactive avatar in learning, cognitive overload might result, which can also affect other learning-related outcomes. In addition, learners differ in interaction in terms of their prior experience and extraneous cognitive load. In the best configuration interaction for experts is present whereby it is absent for learners with low prior experience. At some point interaction might be helpful for experienced learners to faster identify learning content that they do not know in detail, and it helps them to better focus on knowledge that they still need to improve. Lastly, our results demonstrate that it is useful to analyze a target group or context in detail before developing any kind of gamification concept or learning materials. Depending on different kind of criteria, learners differ in how they experience avatars and learning materials. Females are emotionally connected to avatars when they are familiar, when they better understand the meaning of a training, when they are supported in active discovery and intrinsic as well as extrinsic motivation. Men are more emotionally attached to interactive avatars and a training that supports meaning and both kinds of interaction. When we use prior knowledge as subgroups to analyze configurations for extraneous cognitive load, we can observe that interaction is crucial for experts but not for learners with low experience, which is the same with MEA, which is important for experts but not for novices. Besides gender and experience, other aspects can be used to identify and consider different subgroups among a specific context, such as age or the aspect of whom learners want to compete with (Santhanam et al. 2016; Shen et al. 2016). Such aspects need to be carefully analyzed and considered before developing any kind of online learning material or any gamification concept (these observations find support for other elements such as competition - see Santhanam et al. (2016)).

Summarizing the results, our research provides several theoretical as well as practical implications. First, the results of our research demonstrate which conditions are important for designing avatars in learning. Our results have the potential to contribute to different streams of literature. We contribute to learning theory because we can give implications about how to design emotionally attaching avatars. In addition, we contribute to the development of learning material in general because we are able to better explain how online learning materials presented by an avatar can influence a learner's extraneous cognitive load. We contribute to streams of learning theory because we can better explain the role and meaning of the relationship between avatars that are used as tutors or teachers and learners that work with them. Therefore, we can explain how the familiarity (by referring to self-expansion theory) can influence specific outcome variables such as satisfaction or emotional attachment. In addition, the results of our study provide more information about configurations that are important for web-based trainings, such as motivation and aesthetic experience. Furthermore, we can give implications about the relevance of avatar interaction in learning processes and how an interaction between a learner and an avatar can influence extraneous cognitive load, satisfaction with the learning process, and emotional attachment. Finally, we can contribute to literature streams such as gamification or game design because we can provide precise implications regarding design components of avatars. From a practical perspective, our study can provide insights to developers of learning applications or learning-related gamification concepts about what to consider when designing avatars for learning purposes. Seaborn and Fels (2015) claim that more research should focus on the design and meaning of individual game design elements. With this implication in mind, our study can assist practitioners in developing avatars for learning applications.

\section{Future Research, Limitations and Conclusion}

This study is not without limitations that provide implications for future research. This study did not consider the effects of avatar designs on learning outcomes or on a learner's general intention to use an avatar-based learning system more regularly. However, research indicates that emotional attachment, satisfaction, and cognitive load can be important to positively influence learning outcomes (Scaife and Rogers 2001; Witmer and Singer 1998). Future research could develop online trainings with avatars to determine how a specific avatar design can affect learning outcomes by referring to skill-based, procedural, 
or declarative outcomes (Gupta and Bostrom 2013). Such studies could use a quantitative approach by developing hypotheses and testing them with a structural equation model. Such an analysis could also support a more detailed analysis about the relationships of all three outcome variables. This would also allow to analyze some control variables in more detail. Therefore, the results of our study can be used to derive an avatar design that emotionally attaches users, influences their learning process satisfaction, and reduces extraneous cognitive load. Second, more research should focus on analyzing sub-samples of satisfaction with the learning process in relation to different avatar designs. Gender as well as experience with Excel could be used. Studies could also analyze if satisfaction is related to the gender of avatars. Third, other conditions should be considered for the configuration of dependent variables such as faithfulness of technology appropriation or self-efficacy. Finally, more research should analyze if users react differently to female or male avatars. In our study, we could not detect any effects as to whether male or female avatars affect the learner's emotional attachment, satisfaction, or if they help to reduce cognitive load. The goal of our study was to analyze the relevance of avatars in relation to emotional attachment, satisfaction with the learning process, and extraneous cognitive load of learners. With our study, we present different configurations for avatar design conditions as well as web-based training conditions. Online learning, avatars, and gamification still needs to be analyzed in more detail and our results provide new ideas to make more detailed analyses of avatars and constructs relevant for online learning.

\section{Acknowledgements}

Our thanks go to Sissy-Josefina Ernst for her sketches and drawing skills which has given us the basis for the avatar concept. The research presented in this paper was funded by the German Federal Ministry of Education and Research in the context of the project KoLeArn (www.KoLeArn.de), Grant No. 01BE17008A.

\section{References}

Aron, A., Steele, J. L., Kashdan, T. B., and Perez, M. 2006. "When similars do not attract: Tests of a prediction from the self-expansion model," Personal Relationships (13:4), pp. 387-396.

Artino, A. R. 2008. "Cognitive Load Theory and the Role of Learner Experience: An Abbreviated Review for Educational Practitioners," Association for the Advancement of Computing In Education Journal (16:4), pp. 425-439.

Ayres, P., and Youssef, A. (eds.) 2008. Investigating the influence of transitory information and motivation during instructional animations, International Society of the Learning Sciences.

Bandura, A., and Jourden, F. J. 1991. "Self-Regulatory Mechanisms Governing the Impact of Social Comparison on Complex Decision Making," Journal of Personality and Social Psychology (60:6), pp. 941-951.

Bedwell, W. L., Pavlas, D., Heyne, K., Lazzara, E. H., and Salas, E. 2012. "Toward a Taxonomy Linking Game Attributes to Learning: An Empirical Study," Simulation \& Gaming (43:6), pp. 729-760.

Bughin, J., Hazan, E., Lund, S., Dählström, P., Wiesinger, A., and Subramaniam, A. 2018. Skill shift: Automation and the future of the workforce. https://www.mckinsey.com/featured-insights/futureof-work/skill-shift-automation-and-the-future-of-the-workforce. Accessed 10 April 2019.

Burgers, C., Eden, A., van Engelenburg, M. D., and Buningh, S. 2015. "How feedback boosts motivation in play in a brain-training game," Computers in Human Behavior (48), pp. 94-103.

Chini, J. J., Straub, C. L., and Thomas, K. H. 2016. "Learning from avatars: Learning assistants practice physics pedagogy in a classroom simulator," Physical Review Physics Education Research (12:1), p. 10117.

Christy, K. R., and Fox, J. 2014. "Leaderboards in a virtual classroom: A test of stereotype threat and social comparison explanations for women's math performance," Computers \& Education (78), pp. 66-77.

Christy, K. R., and Fox, J. 2016. "Transportability and presence as predictors of avatar identification within narrative video games," Cyberpsychology, Behavior, and Social Networking (19:4), pp. 283287. 
Deci, E. L., Ryan, R. M., Gagne, M., Leone, D. R., Usunov, J., and Kornazheva, B. P. 2001. "Need Satisfaction, Motivation, and Well-Being in the Work Organizations of a Former Eastern Bloc Country: A Cross-Cultural Study of Self-Determination," Personality and Social Psychology Bulletin (27:8), pp. 930-942.

Derrick, D. C., Jenkins, J. L., and Nunamaker Jr, J. F. 2011. "Design principles for special purpose, embodied, conversational intelligence with environmental sensors (SPECIES) agents," AIS Transactions on Human-Computer Interaction (3:2), pp. 62-81.

Deterding, S., Sicart, M., Nacke, L., O'Hara, K., and Dixon, D. 2011. “Gamification: Using Game Design Elements in Non-Gaming Contexts," Human Factors in Computing Systems, pp. 2425-2428.

Eccles, J. S., and Wigfield, A. 1995. "In the mind of the achiever: The structure of adolescents' academic achievement related-beliefs and self-perceptions," Personality and Social Psychology Bulletin (21:3), pp. 215-225.

Eom, S. B. 2014. “Understanding e-learners' satisfaction with learning management systems," Bulletin of the IEEE Technical Committee on Learning Technology (16:2), p. 3.

Ernst, S.-J., Janson, A., Söllner, M., and Leimeister, J. M. 2016. “It’s about Understanding Each Other's Culture - Improving the Outcomes of Mobile Learning by Avoiding Culture Conflicts," International Conference on Information Systems (ICIS), Dublin, Ireland.

Felder, R. M., and Brent, R. 2005. “Understanding student differences,” Journal of engineering education (94:1), pp. 57-72.

Ferguson, J. M., and DeFelice, A. E. 2010. "Length of online course and student satisfaction, perceived learning, and academic performance," The International Review of Research in Open and Distributed Learning (11:2), pp. 73-84.

Fisher, C. D. 2003. "Why do lay people believe that satisfaction and performance are correlated? Possible sources of a commonsense theory," Journal of Organizational Behavior: The International Journal of Industrial, Occupational and Organizational Psychology and Behavior (24:6), pp. 753-777.

Fiss, P. C. 2011. "Building better causal theories: A fuzzy set approach to typologies in organization research," Academy of Management Journal (54:2), pp. 393-420.

Fornell, C., and Larcker, D. F. 1981. "Evaluating structural equation models with unobservable variables and measurement error," Journal of Marketing research, pp. 39-50.

Greckhamer, T., Misangyi, V. F., and Fiss, P. C. 2013. "The two QCAs: From a small-N to a large-N set theoretic approach," in Configurational theory and methods in organizational research, P. C. Fiss, B. Cambré and A. Marx (eds.): Emerald Group Publishing, pp. 49-75.

Greenberg, B. S., Sherry, J., Lachlan, K., Lucas, K., and Holmstrom, A. 2010. "Orientations to video games among gender and age groups," Simulation \& Gaming (41:2), pp. 238-259.

Gupta, S., and Bostrom, R. 2013. "Research Note-An Investigation of the Appropriation of TechnologyMediated Training Methods Incorporating Enactive and Collaborative Learning," Information Systems Research (24:2), pp. 454-469.

Gupta, S., and Bostrom, R. P. 2009. "Technology-mediated learning: A comprehensive theoretical model," Journal of the Association for Information Systems (10:9), pp. 686-714.

Gupta, S., Bostrom, R. P., and Huber, M. 2010. "End-user training methods: What we know, need to know," ACM SIGMIS Database: the DATABASE for Advances in Information Systems (41:4), pp. 939.

Hailikari, T., Katajavuori, L., and Lindblom-Ylanne, S. 2008. "The relevance of prior knowledge in learning and instructional design," American journal of pharmaceutical education (72:5), p. 113.

Hazan, C., and Zeifman, D. 2008. "Pair bonds as attachments," in Handbook of attachment: Theory, research, and clinical applications, J. Cassidy and P. R. Shaver (eds.), New York: Guilford Press, pp. 336-354.

Hoffner, C., and Buchanan, M. 2005. "Young adults' wishful identification with television characters: The role of perceived similarity and character attributes," Media Psychology (7:4), pp. 325-351. 
Hui, W., Hu, P.-H., Clark, T. H. K., Tam, K. Y., and Milton, J. 2008. “Technology-assisted learning: A longitudinal field study of knowledge category, learning effectiveness and satisfaction in language learning," Journal of Computer Assisted Learning (24:3), pp. 245-259.

Jia, Y., Xu, B., Karanam, Y., and Voida, S. (eds.) 2016. Personality-targeted gamification: A survey study on personality traits and motivational affordances, ACM.

Kim, Y. J., Baker, J., and Song, J. 2007. “An Exploratory Study of Social Factors Influencing Virtual Community Members' Satisfaction with Avatars," Communications of the Association for Information Systems (20), pp. 567-593.

Lee, J. J., Ceyhan, P., Jordan-Cooley, W., and Sung, W. 2013. "GREENIFY: A Real-World Action Game for Climate Change Education," Simulation \& Gaming (44:2-3), pp. 349-365.

Lee, K. C., and Kwon, S. 2008. "A cognitive map-driven avatar design recommendation DSS and its empirical validity,” Decision Support Systems (45:3), pp. 461-472.

Lin, L., Atkinson, R. K., Christopherson, R. M., Joseph, S. S., and Harrison, C. J. 2013. "Animated agents and learning: Does the type of verbal feedback they provide matter?” Computers \& Education (67), pp. 239-249.

Marchand, G. C., and Gutierrez, A. P. 2012. "The role of emotion in the learning process: Comparisons between online and face-to-face learning settings," The Internet and Higher Education (15:3), pp. 150-160.

Mattingly, B. A., and Lewandowski, G. 2013. "The power of one: Benefits of individual self-expansion," The Journal of Positive Psychology (8:1), pp. 12-22.

Mayer, R. E., and Moreno, R. 2003. "Nine ways to reduce cognitive load in multimedia learning," Educational Psychologist (38:1), pp. 43-52.

Means, B., Toyama, Y., Murphy, R., Bakia, M., and Jones, K. 2009. "Evaluation of evidence-based practices in online learning: A meta-analysis and review of online learning studies," US Department of Education.

Mull, I., Wyss, J., Moon, E., and Lee, S.-E. 2015. "An exploratory study of using 3D avatars as online salespeople," Journal of Fashion Marketing and Management: An International Journal (19:2), pp. 154-168.

Noels, K. A. 2001. "New orientations in language learning motivation: Towards a model of intrinsic, extrinsic, and integrative orientations and motivation," Motivation and second language acquisition (23), pp. 43-68.

Paas, F., Renkl, A., and Sweller, J. 2003. "Cognitive Load Theory and Instructional Design: Recent Developments," Educational Psychologist (38:1), pp. 1-4.

Pérez-Marín, D., and Pascual-Nieto, I. 2013. "An exploratory study on how children interact with pedagogic conversational agents," Behaviour \& Information Technology (32:9), pp. 955-964.

Pfeuffer, N., Benlian, A., Gimpel, H., and Hinz, O. 2019. "Anthropomorphic Information Systems," Business \& Information Systems Engineering, pp. 1-11.

Pintrich, P. R. 2003. "A motivational science perspective on the role of student motivation in learning and teaching context," Journal of Educational Psychology (95:4), pp. 667-689.

Poonam, D. C. 1997. "Intrinsic motivation and academic achievement: What does their relationship imply for the classroom teacher?" Remedial and special education (18:1), pp. 12-19.

Ragin, C. C. 2009. "Qualitative comparative analysis using fuzzy sets (fsQCA)," Configurational comparative methods: Qualitative comparative analysis (QCA) and related techniques (51), pp. 87121.

Santhanam, R., Liu, D., and Shen, Wei-Cheng, Milton 2016. "Research Note-Gamification of TechnologyMediated Training: Not All Competitions are the Same," Information Systems Research (27:2), pp. $453-465$. 
Scaife, M., and Rogers, Y. 2001. "Informing the design of a virtual environment to support learning in children," International Journal of human-computer studies (55:2), pp. 115-143.

Schmidt-Kraepelin, M., Thiebes, S., Tran, M. C., and Sunyaev, A. 2018. "What's in the Game? Developing a Taxonomy of Gamification Concepts for Health Apps," Hawaii International Conference on System Science (HICSS).

Schneider, C. Q., and Wagemann, C. 2012. Set-theoretic Methods for the Social Sciences: A Guide to Qualitative Comparative Analysis, Cambridge: Cambridge University Press.

Schöbel, S., and Janson, A. 2018. "Is it all about Having Fun? - Developing a Taxonomy to Gamify Information Systems," European Conference on Information Systems (ECIS).

Scott, M., Pereira, L., and Oakley, I. 2014. "Show me or tell me: Designing avatars for feedback," Interacting with computers (27:4), pp. 458-469.

Seaborn, K., and Fels, D. I. 2015. "Gamification in theory and action: A survey," International Journal of human-computer studies (74), pp. 14-31.

Shen, W.-C. M., Liu, D., Santhanam, R., and Evans, D. A. 2016. "Gamified Technology-Mediated Learning: The Role of Individual Differences,” Pacific Asia Conference on Information Systems (PACIS).

Shute, V. J. 2008. "Focus on formative feedback," Review of educational research (78:1), pp. 153-189.

Steed, A., Pan, Y., Zisch, F., and Steptoe, W. (eds.) 2016. The impact of a self-avatar on cognitive load in immersive virtual reality, IEEE.

Suh, A., Cheung, C. M. K., Ahuja, M., and Wagner, C. 2017. "Gamification in the Workplace: The Central Role of the Aesthetic Experience,” Journal of Management Information Systems (34:1), pp. 268305.

Suh, K.-S., Kim, H., and Suh, E. K. 2011. "What if your Avatar looks like you? Dual Congruity Perspectives for Avatar User," MIS Quarterly (35:3), pp. 711-729.

Sun, H., and Ping, Z. 2006. "The role of moderating factors in user technology acceptance," International Journal of human-computer studies (64:2), pp. 53-78.

Super, J., Keller, R. H., Betts, T. K., and Roach Humphreys, J. (eds.) 2019. Simulation Games: Learning Goal Orientations and Norms for Knowledge Sharing, Academy of Management Briarcliff Manor, NY 10510.

Sweller, J. 1994. “Cognitive load theory, learning difficulty, and instructional design,” Learning and Instruction (4:4), pp. 295-312.

Thiebes, S., Lins, S., and Basten, D. 2014. "Gamifying Information Systems: A Synthesis of Gamification Mechanics and Dynamics," European Conference on Information Systems (ECIS).

Thomson, M., MacInnis, D. J., and Park, C. W. 2005. "The ties that bind: Measuring the strength of consumers' emotional attachments to brands," Journal of consumer psychology (15:1), pp. 77-91.

Van Gerven,, P. W. M., Paas,, F. G. W. C., Van Merrienboer,, J. J. G., and Schmidt, H. G. 2002. “Cognitive load theory and aging: effects of worked examples on training efficiency," Learning and Instruction (12), pp. 87-105.

van Merriënboer, J. J. G., and Sweller, J. 2005. "Cognitive Load Theory and Complex Learning: Recent Developments and Future Directions," Educational Psychology Review (17:2), pp. 147-177.

Venkatesh, V., and Morris, M. G. 2000. "Why don't men ever stop to ask for directions? Gender, social influence, and their role in technology acceptance and usage behavior," MIS Quarterly (24:1), pp. $115^{-139 .}$

Witmer, B. G., and Singer, M. J. 1998. "Measuring presence in virtual environments: A presence questionnaire," Presence: Teleoperators and virtual environments (7:3), pp. 225-240. 